\title{
Stability of Amoxicillin and Clavulanic Acid in Separate Containers for Administration via a Y-Site
}

\author{
Sarah Fawaz \\ Mahboub Merzouk (D) \\ Stephen Barton (D) \\ Shereen Nabhani-Gebara \\ Faculty of Science, Engineering and \\ Computing, Kingston University, \\ London, UK
}

\begin{abstract}
Rationale: With the discovery of new antibiotics diminishing, optimising the administration of existing antibiotics such as amoxicillin-clavulanic acid has become a necessity. At present, the optimal approach for enhancing the effectiveness of time-dependent antibiotics involves extending the time at which antibiotic concentrations are maintained above the minimal inhibitory concentration by prolonging the infusion time. This pharmacodynamic rationale cannot be applied to co-amoxiclav because of poor stability at room temperature. The aim of this study was to establish the shelf-life of amoxicillin and clavulanic acid prepared in separate containers to determine the feasibility of 24-hr continuous infusion therapy.
\end{abstract}

Methods: A previously developed and validated stability-indicating HPLC method was used to establish the shelf-life of reconstituted amoxicillin and clavulanic acid when prepared in separate containers. Stability at clinical concentration was evaluated at three temperatures. To establish whether there were significant differences at the level of both active ingredients and temperature, results were analysed using analysis of covariance (ANCOVA) to assess differences between the attained slopes of regression.

Results: Data obtained indicated amoxicillin and clavulanic acid stability superior to that previously proposed making it suitable for continuous infusion therapy. Analysis of regression slopes via ANCOVA showed that temperature significantly affected amoxicillin and clavulanic acid stability. Amoxicillin retained $90 \%$ of its initial concentration for $80.3 \mathrm{hrs}$ when stored at $4{ }^{\circ} \mathrm{C}, 24.8 \mathrm{hrs}$ at $25^{\circ} \mathrm{C}$ and $9 \mathrm{hrs}$ when incubated at $37^{\circ} \mathrm{C}$. Clavulanic acid retained $90 \%$ of its initial concentration for $152 \mathrm{hrs}$ when stored at $4{ }^{\circ} \mathrm{C}, 26 \mathrm{hrs}$ at $25^{\circ} \mathrm{C}$ and $6.4 \mathrm{hrs}$ when incubated at $37^{\circ} \mathrm{C}$.

Conclusion: Amoxicillin and clavulanic acid are suitable for administration via continuous infusion when prepared, stored, and administered in separate containers. Results obtained from this study aid in ameliorating current dosing regimens to optimise antibiotic efficacy; however, more in-depth amoxicillin and clavulanic acid y-site compatibility studies are warranted.

Keywords: continuous infusion, amoxicillin, clavulanic acid and antibiotic resistance

\section{Introduction}

Despite advances in modern medicine, antibiotic dosing regimens have remained largely unchanged since their discovery. Previously, antibiotic dosing schedules were empirically designed based on in vitro data and clinical experience. By failing to encompass characteristics including dose response, dosing interval, optimal duration of therapy or post-antibiotic effects, dosing regimens based on an understanding of pharmacodynamics (PD) were therefore not established. ${ }^{1,2}$

The rise of antimicrobial resistance has prompted investigation into optimising the administration of antibiotics currently used in practice. $\beta$-lactams are the most extensively utilised antibiotics due to their relatively high effectiveness, low cost,
Correspondence: Sarah Fawaz Email s.fawaz@kingston.ac.uk 
ease of delivery and minimal side effects. Currently, parenteral administration of $\beta$-lactam antibiotics is via bolus dosing which produces unnecessary erratic peak plasma and low trough concentrations below the minimal inhibitory concentration (MIC) between dosing intervals. ${ }^{3}$ It has been established that maintaining serum concentrations above the MIC of the respective organism for $\geq 50 \%$ of the dosing interval promotes maximal PD activity. ${ }^{4,5}$

Amoxicillin is widely used for the treatment of uncomplicated penicillin-sensitive infections; however, its use alone is limited as beta-lactamase producing bacteria can easily destroy it. Concomitant administration with clavulanic acid broadens the antibacterial spectrum by exerting a pronounced synergistic effect. ${ }^{6}$

Administration regimens including more frequent dosing or continuous infusion have been found to optimise the PD profile of amoxicillin. Furthermore, continuous infusion of $\beta$-lactam antibiotics has demonstrated a reduction in the total daily dose of drug required, ${ }^{3}$ shorter treatment duration, $^{7}$ as well as a reduction in the formation of resistant bacteria. ${ }^{3,8,9}$ Although administration by continuous infusion maximises $\beta$-lactam's PD properties, uncertainty regarding amoxicillin's stability after reconstitution and dilution presents a challenge in practice when assigning a shelf-life to infusion solutions. ${ }^{10-12}$

The literature suggests that amoxicillin and clavulanic acid undergo hydrolytic degradation after reconstitution. ${ }^{11,13}$ Kinetic studies have reported the catalytic effect of clavulanic acid on amoxicillin. ${ }^{14,15}$ The two species, and possibly their decomposition products, interact in solution where an enhancement of the catalytic effect of one reacting species upon another due to proportional increased concentration of the catalyst is likely. ${ }^{14}$ The catalysis of amoxicillin by clavulanic acid or vice versa in infusion solutions prompts further investigation.

To expand the breadth of current knowledge, a proposed strategy for enhancing amoxicillin and clavulanic acids stability involves the preparation and administration of the parenteral amoxicillin and clavulanic acid via separate infusion devices. Little is known about the physiochemical stability of amoxicillin and clavulanic acid alone in comparison to the combination of amoxicillin with clavulanic acid infusion solutions at clinically relevant concentrations. The logistical advantages of simultaneous administration in separate devices (eg, improved stability), will pave the way for optimising current treatment strategies (ie, via concurrent Y-site administration) which have potential to improve and enhance clinical efficacy.

\section{Materials and Methods Materials}

Amoxicillin sodium, potassium clavulanate and caffeine reference standards were purchased from Sigma Aldrich, as were ammonium acetate and glacial acetic acid. Water for injection (WFI) was purchased from The Pharmacy, Kingston upon Thames, UK. Methanol (HPLC grade) and acetonitrile (HPLC grade) were purchased from VWR and deionised water was generated in the laboratory at Kingston University, London, UK.

\section{Instrumentation}

Qualitative analysis of amoxicillin-clavulanic acid was carried out using $600 \mathrm{mHz}$ base frequency Bruker Advance III Two-channel FT-NMR spectrometer.

Quantitative analysis of amoxicillin-clavulanic acid was carried out using an Agilent 1260 HPLC system with single wavelength UV detection and ChemStation software.

\section{Qualitative NMR Investigation}

Preliminary examinations of amoxicillin, clavulanic acid and co-amoxiclav were carried out by the means of NMR spectroscopy to provide an indication of how fast they degraded and inform the development of the quantitative high performance liquid chromatography (HPLC) stability indicating method (SIM). Amoxicillin, clavulanic acid and co-amoxiclav solution at the relevant concentrations were prepared and transferred into NMR tubes. The instrument was programmed to periodically analyze throughout a $72 \mathrm{hr}$ period using a $1 \mathrm{D}$ (proton) $\mathrm{H}$ NMR using the "noesyld presaturation" water suppression method.

\section{HPLC SIM}

To quantify amoxicillin, clavulanic acid and co-amoxiclav concentrations with respect to time, HPLC was used. A previously developed and validated method (in accordance with ICH guidelines) was utilised. ${ }^{11}$ Separation was conducted using Phenomenex Spherisorb $5 \mu \mathrm{m}$, $100 \times 4.6 \mathrm{~mm}$ column with a binary mobile phase composition consisting of Ammonium Acetate (5mM) pH4 and acetonitrile $(50: 50 \mathrm{v} / \mathrm{v})$. Analysis was performed at detection wavelength $215 \mathrm{~nm}$. Flow rate was $0.5 \mathrm{~mL} / \mathrm{min}^{11}$ 
The calibration curves obtained for amoxicillin (range $=0-667 \mathrm{ppm}$ ) and clavulanic acid (range $=0-133 \mathrm{ppm}$ ) demonstrated good linearity by which the representative linear equations were $\mathrm{y}=1.0001 \mathrm{x}+2.3058\left(\mathrm{R}^{2}=0.999\right)$ and $\mathrm{y}=1.0066 \mathrm{x}-0.0397\left(\mathrm{R}^{2}=0.999\right)$, respectively.

\section{Quantitative HPLC Assay}

In clinical settings, a $1.2 \mathrm{~g}$ amoxicillin-clavulanic acid vial is reconstituted with $20 \mathrm{~mL}$ WFI and is diluted further with $50 \mathrm{~mL}$ of diluent $(1200 \mathrm{mg} / 70 \mathrm{~mL}=17143 \mathrm{ppm})$. To mimic these conditions, $1 \mathrm{~g}$ of amoxicillin sodium and $0.2 \mathrm{~g}$ of potassium clavulanate were accurately weighed and transferred into separate containers. Amoxicillin and clavulanic acid were each reconstituted using $70 \mathrm{~mL}$ WFI. The two solutions were each split into three subsamples and stored at the relevant temperature condition. Noteworthy is the concentrations may vary with differing dilution practices. The concentration used in this study falls within the higher range of clinical concentrations, therefore, the stability of more dilute solutions, commonly used in practice, are expected to exhibit lower degradation rates (ie, higher stability).

To evaluate preparation and storage feasibility, the stability of solutions was assessed at $4^{\circ} \mathrm{C}$. Ambient and $37^{\circ} \mathrm{C}$ temperature conditions were selected in order to mimic average and high temperatures experienced in hospital wards due to seasonal variations. Sampling was undertaken at 2, 4, 6, 8, $10,12,24,30,36,50,56$, and $152 \mathrm{hrs}$ and the infusion solution was considered stable while the percentage recovery of amoxicillin and clavulanic acid remained above $90 \%$.

\section{Data and Statistical Analysis}

Raw data obtained was corrected for the internal standard. Results are reported as the residual ratio of amoxicillin and clavulanic acid concentration from three replicates. To determine whether amoxicillin or clavulanic acid concentration decreased significantly over time, the slope of the linear regression line for each condition was tested against the null hypothesis $\left(\mathrm{H}_{0}=\right.$ no deviation from zero) using a one tailed $t$-test at the $99 \%$ level of significance. To assess whether there was a significant difference between amoxicillin and clavulanic acids rate of degradation, results were analysed using analysis of variance (ANOVA) at the 95\% level of significance. One tailed $t$-tests and ANOVA analysis was undertaken using Microsoft Excel 365. To establish whether there were significant differences at the level of temperature, results were analysed using analysis of covariance (ANCOVA) at the $75 \%$ level of significance, to assess differences between the attained slopes of regression. ANCOVA analysis was undertaken using IBM SPSS 26.

\section{Results NMR}

The preliminary NMR method for studying amoxicillin, clavulanic acid and co-amoxiclav degradation, detected a considerable difference in the rate at which degradation occurs in separate solutions compared to the combined solution. The spectra relating to the combined solution (after 72 hours of dilution) in Figure 1, displays the appearance of new peaks, most prominently in circled regions, suggesting that the rate of degradation is accelerated when amoxicillin and clavulanic acid are prepared in combination. Qualitative data was retrieved from NMR as this technique lacks sensitivity and suffers from signal overlapping when analysing mixtures.

\section{HPLC}

Amoxicillin and clavulanic acid solutions retained more of their initial concentration for longer than reported in previously published results where they were prepared in combination. ${ }^{11}$ The slopes of the regression lines for both active pharmaceutical ingredients (APIs) at the three temperatures studied showed significant deviation from zero at the $99 \%$ level of confidence, indicating amoxicillin and clavulanic acid exhibit degradation with time (Table 1). Similarly, to previously published data, ${ }^{11}$ ANCOVA showed that solutions stored at lower temperatures remained stable for significantly longer than solutions at higher temperatures (Table 2). The influence of temperature of both APIs is displayed in Figure 2.

Stability data for all studied concentrations are displayed in Table 1. Amoxicillin retained $90 \%$ of its initial concentration for $80.3,24.8$ and 9 hours at $4^{\circ} \mathrm{C}, 25^{\circ} \mathrm{C}$ and $37^{\circ} \mathrm{C}(\%$ recovery at 6 hours $=95 \%$ and at 8 hours $=95 \%$ at $25^{\circ} \mathrm{C}$ ), respectively. Clavulanic acid retained $90 \%$ of initial concentration for 152,26 and 6.4 hours at $4^{\circ} \mathrm{C}$, $25^{\circ} \mathrm{C}$ and $37^{\circ} \mathrm{C}$ (\%recovery at 6 hours $=96 \%$ and at 8 hours $=98 \%$ at $25^{\circ} \mathrm{C}$ ), respectively. Significant differences between regression slopes of temperature conditions for each API were observed (Table 1).

\section{Discussion}

To the best of our knowledge, this is the first study of its kind to concomitantly examine amoxicillin and clavulanic 


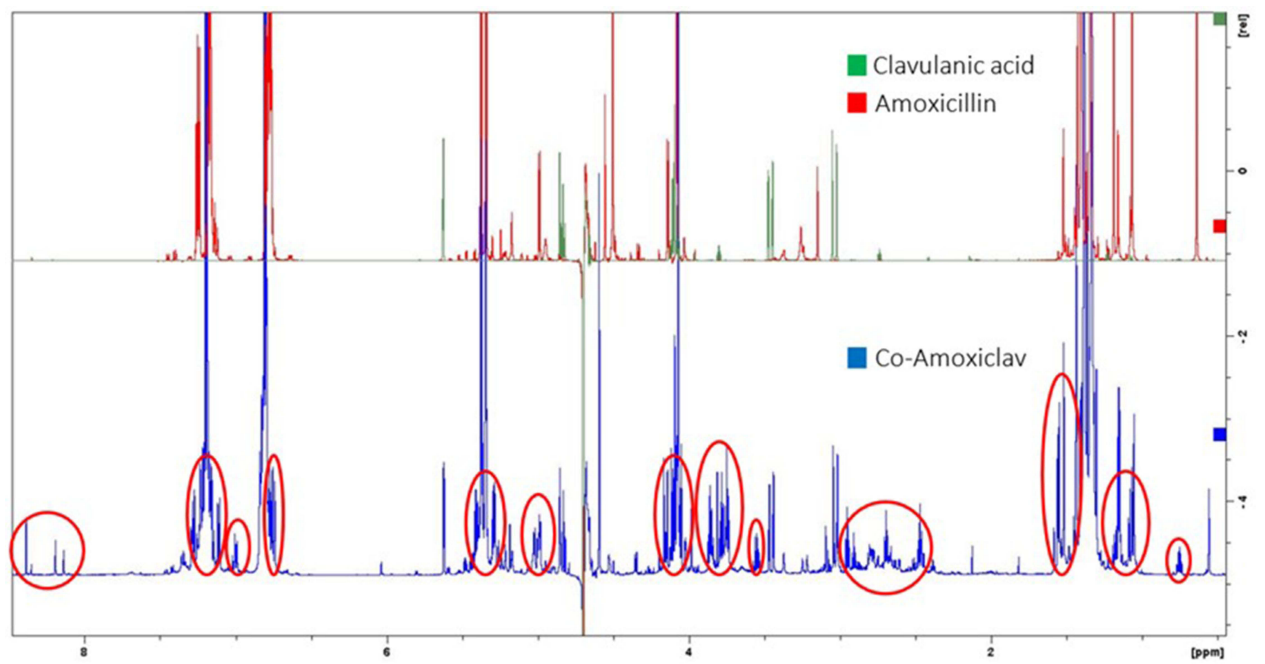

Figure I Preliminary NMR analysis of amoxicillin, clavulanic acid and co-amoxiclav after 72 hours of reconstitution. Red circles highlighting the increase in peak intensities, suggesting co-amoxiclav (combination of both compounds) degradation.

acid stability at clinical concentrations across a range of temperatures. The consequent understanding of the molecular stability of amoxicillin and clavulanic acid in separate solutions allows for a greater evaluation of its suitability for administration via prolonged and continuous infusion, aiding in the development of novel treatment strategies. Amoxicillin and clavulanic acid have not previously been considered for separate continuous infusion when administered in combination, however, findings from this study demonstrate its feasibility.

The methods of antibiotic administration have remained unchanged since their discovery. This poses the question of whether antibiotics are being used to their greatest potential? Dosing regimens were established from data describing in vitro antibacterial activity as well as clinical experience, thus leading to intermittent bolus dosing. These regimens were infrequently validated yet have been standard clinical procedure for numerous decades. Therefore, it is time for re-evaluating conventional practice. Current administration methods should be challenged based on the advanced knowledge of the pharmacodynamics of these antibiotics.

Effective antibiotic treatment modalities mainly consist of two variables: the dose and duration of treatment. Drug efficiency studies have determined the effective dose and duration for co-amoxiclav treatment regimen, although, a limitation of this approach is that it only provides information for the regimen being analysed and offers no indication for other potential dosing regimens. Traditional antibiotic dosing consists of administering a fixed dose for a specified duration. However, the increase in antibiotic resistant bacteria poses a threat to the effectiveness in treating bacterial infections. ${ }^{16}$ With the lack of new antibiotics and the increase in antibiotic resistance,

Table I Displaying the Linear Regression Equations for Amoxicillin and Clavulanic Acid Conditions Used to Calculate the Predicted Time at Which Residual Ratio of Amoxicillin Falls Below 90\%

\begin{tabular}{|l|c|c|c|}
\hline Condition & Deviation of Slope from Zero & Linear Equation & Predicted Stability (hrs) \\
\hline Amoxicillin $4{ }^{\circ} \mathrm{C}$ & Significant $(p<0.000 I)$ & $y=-0.1419 x+10 I .4$ & 80.3 \\
Amoxicillin $25^{\circ} \mathrm{C}$ & Significant $(p<0.000 I)$ & $y=-0.33 I 5 x+98.2 I$ & 24.8 \\
Amoxicillin $37^{\circ} \mathrm{C}$ & Significant $(p<0.000 I)$ & $y=-1.014 x+99.02$ & 9.0 \\
Clavulanic Acid $4{ }^{\circ} \mathrm{C}$ & Significant $(p<0.000 I)$ & $y=-0.07 I 80 x+100.3$ & 152 \\
Clavulanic Acid $25^{\circ} \mathrm{C}$ & Significant $(p<0.000 I)$ & $y=-0.3162 x+98.34$ & 26.4 \\
Clavulanic Acid $37^{\circ} \mathrm{C}$ & Significant $(p<0.000 I)$ & $y=-1.2727 x+98.166$ & 6.4 \\
Combination $4^{\circ} \mathbf{C}^{\prime \prime}$ & Significant $(p<0.000 I)$ & $y=-1.26 I x+102.1$ & 9.6 \\
Combination $25^{\circ} \mathrm{C}^{\prime \prime}$ & Significant $(p<0.000 I)$ & $y=-1.438 x+100.8$ & 7.5 \\
Combination $37^{\circ} \mathrm{C}^{\prime \prime}$ & Significant $(p<0.000 I)$ & $y=-2.449 x+101.0$ & 4.5 \\
\hline
\end{tabular}

Notes: Previously Reported Predicted Stability Data for Co-Amoxiclav is Displayed in BOLD. ' 
Table 2 Results of ANCOVA Analyses Performed at the Level of Active Ingredient at the $75 \%$ Confidence Level

\begin{tabular}{|c|c|c|}
\hline $\begin{array}{l}\text { Active } \\
\text { Ingredients }\end{array}$ & $\begin{array}{l}\text { ANCOVA (All } \\
\text { Temperature } \\
\text { Conditions) }\end{array}$ & $\begin{array}{c}\text { ANCOVA (Individual } \\
\text { Analyses) }\end{array}$ \\
\hline Amoxicillin & $(p \leq 0.001)$ & $\begin{array}{l}4^{\circ} \mathrm{C} \text { vs } 25^{\circ} \mathrm{C}:(\mathrm{p} \leq 0.00 \mathrm{I}) \\
4^{\circ} \mathrm{C} \text { vs } 37^{\circ} \mathrm{C}:(\mathrm{p} \leq 0.00 \mathrm{I}) \\
25^{\circ} \mathrm{C} \text { vs } 37^{\circ} \mathrm{C}:(\mathrm{p}=0.020)\end{array}$ \\
\hline $\begin{array}{l}\text { Clavulanic } \\
\text { Acid }\end{array}$ & $(p<0.00 I)$ & $\begin{array}{l}4^{\circ} \mathrm{C} \text { vs } 25^{\circ} \mathrm{C}:(\mathrm{p}<0.001) \\
4^{\circ} \mathrm{C} \text { vs } 37^{\circ} \mathrm{C}:(\mathrm{p}<0.00 \mathrm{I}) \\
25^{\circ} \mathrm{C} \text { vs } 37^{\circ} \mathrm{C}:(\mathrm{p}=0.010)\end{array}$ \\
\hline
\end{tabular}

strategies to improve the utility of existing antibiotics, like co-amoxiclav, are mandatory. ${ }^{17}$

Finding optimal treatment regimens is critical in ensuring the prolonged effectiveness of antibiotics like coamoxiclav. Optimising amoxicillin and clavulanic acids potential for successful clinical outcomes requires consideration of $\mathrm{PD}$ attributes to maximise bacterial eradication as well as minimise the capability for further resistance. If traditional dosing regimens are modified to deliver amoxicillin and clavulanic acid doses where concentrations are maintained above the MIC of infecting organism for $50 \%$ of the dosing interval then the initial facilitation of resistant bacteria will disappear. ${ }^{16}$

Even though clavulanic acid is not available in pharmaceutical formulation and is not licensed to be administered alone, this study paves the way for innovation to overcome stability concerns. Data obtained indicated stability superior to that previously proposed ${ }^{6,11,13,18,19}$ rendering it suitable for extended or continuous infusion therapy. Results obtained are in alignment with those recently published, suggesting that storage temperature significantly influences the stability of amoxicillin and clavulanic acid (Tables 1-3). ${ }^{11}$

Studies have previously confirmed that there is no difference between the pharmacokinetic data derived from serum level determinations of amoxicillin or clavulanic acid after administration of the single substances or of the combination. ${ }^{20}$ Therefore, simultaneous administration of amoxicillin and clavulanic acid as single substances via a y-site would improve the effectiveness of therapy without altering the dose or dosing schedule, giving no increase in toxicity. ${ }^{11}$

Stability data should be specific to in-use conditions and the dosing regimen, thus, clinically relevant
A Amoxicillin and Clavulanic Acid Stability after Reconstitution

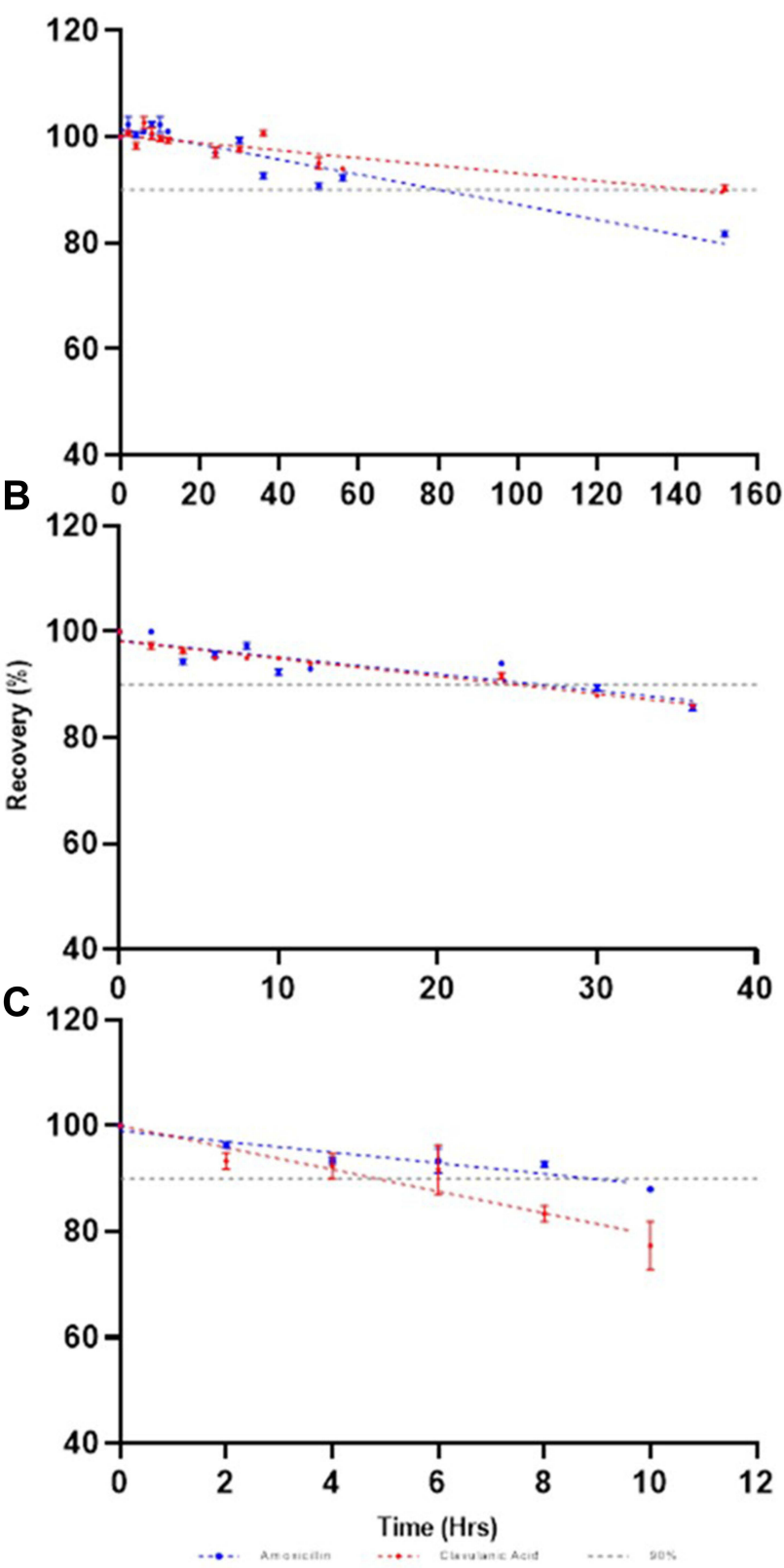

Figure 2 Stability of amoxicillin and clavulanic acid over time at (A) $4^{\circ} \mathrm{C}$, (B) $25^{\circ} \mathrm{C}$ and (C) $37^{\circ} \mathrm{C}$ : mean \% of intact molecule as a function of time. Error bars: \pm standard deviation.

concentrations, diluent, and temperatures of solutions during storage and administration were studied to establish shelf-life of amoxicillin and clavulanic acid after reconstitution and dilution. Compatibility study carried out, including visual inspection for precipitation when two solutions come in contact with each other showed no changes solution colour or clarity, however, more in-depth y-site compatibility studies are warranted. 
Table 3 Results of One-Tailed $t$-Tests at the $99 \%$ Confidence Level and ANOVA Analyses Performed at the Level of Temperature at the $95 \%$ Confidence Level

\begin{tabular}{|l|c|c|}
\hline \multirow{2}{*}{ Temperature } & \multicolumn{2}{|c|}{ Amoxicillin vs Clavulanic Acid } \\
\cline { 2 - 3 } & One-Tailed $t$-Test & ANOVA \\
\hline $4^{\circ} \mathrm{C}$ & $(p=0.292)$ & $(p=0.582)$ \\
$25^{\circ} \mathrm{C}$ & $(p=0.429)$ & $(p=0.858)$ \\
$37^{\circ} \mathrm{C}$ & $(p=0.144)$ & $(p=0.280)$ \\
\hline
\end{tabular}

\section{Conclusion}

This study demonstrates that amoxicillin and clavulanic acid demonstrate stability for longer than that stated by manufacturers and previous stability studies when prepared as separate solutions. Results obtained suggest amoxicillin and clavulanic acids shelf-life is longer when they are prepared as separate solutions, rendering it suitable for administration via continuous infusion or for outpatient settings in terms of stability. Multifactor statistical analysis indicated that the stability of both APIs was significantly influenced by storage temperature. Findings from this study aid in improving current dosing regimens to optimise amoxicillin and clavulanic acid efficacy.

\section{Funding}

This research did not receive any specific grant from funding agencies in the public, commercial, or for notfor-profit sectors.

\section{Disclosure}

The authors report no conflicts of interest in this work.

\section{References}

1. Carbón C. Single-dose antibiotic therapy: what has the past taught us? J Clin Pharmacol. 1992;32:686-691. doi:10.1002/j.1552-4604.1992.tb03870.x

2. Vincent J-L. Intensive Care Medicine: Annual Update 2002. Springer Science \& Business Media; 2003.

3. Rello J, Kollef MH, Díaz E, Rodríguez A. Infectious Diseases in Critical Care. Springer Science \& Business Media; 2010.

4. Turnidge JD. The pharmacodynamics of $\beta$-Lactams. Clin Infect Dis. 1998;27(1):10-22. doi:10.1086/514622

Drug Design, Development and Therapy

\section{Publish your work in this journal}

Drug Design, Development and Therapy is an international, peerreviewed open-access journal that spans the spectrum of drug design and development through to clinical applications. Clinical outcomes, patient safety, and programs for the development and effective, safe, and sustained use of medicines are a feature of the journal, which has also
5. Craig WA. State-of-the-art clinical article: pharmacokinetic/pharmacodynamic parameters: rationale for antibacterial dosing of mice and men. Clin Infect Dis. 1998;26:1-10. doi:10.1086/516284

6. Vega EM, Manzo RH, Sola N. Improving the stability of potassium clavulanate in admixture with amoxicillin. Hosp Pharm. 2008; 15:183-185.

7. Macgowan AP, Bowker KE. Continuous infusion of $\beta$-lactam antibiotics. Clin Pharmacokinet. 1998;35:391-402. doi:10.2165/ 00003088-199835050-00004

8. Young RJ, Lipman J, Gin T, Gomersall CD, Joynt GM, Oh TE. Intermittent bolus dosing of ceftazidime in critically ill patients. J Antimicrob Chemother. 1997;40:269-273. doi:10.1093/jac/40.2.269

9. Fantin B, Farinotti R, Thabaut A, Carbon C. Conditions for the emergence of resistance to cefpirome and ceftazidime in experimental endocarditis due to pseudomonas aeruginosa. J Antimicrob Chemother. 1994;33:563-569. doi:10.1093/jac/33.3.563

10. Fawaz S, Barton S, Whitney L, Swinden J, Nabhani-Gebara S. Stability of meropenem after reconstitution for administration by prolonged infusion. Hosp Pharm. 2018;54:1-7. doi:10.1177/ 0018578718779009

11. Fawaz S, Dixon B, Barton S, Mohamed A, Nabhani-Gebara S. Suitability of amoxicillin-clavulanic acid for administration via prolonged infusion. Drug Des Devel Ther. 2020;14:103-109. doi:10.2147/DDDT.S230459

12. Sabbagh Dit Hawasli R, Barton S, Nabhani-Gebara S. Ambulatory chemotherapy: past, present, and future. $J$ Oncol Pharm Pract. 2021;107815522098591. doi:10.1177/1078155220985916

13. Carlier M, Verstraete AG, De Waele JJ, Stove V. Stability of amoxicillin and amoxicillin/clavulanic acid reconstituted in isotonic saline. $J$ Chemother. 2017;29(1):54-56. doi:10.1179/1973947815y.00000 00052

14. Vahdat L, Sunderland VB. Kinetics of amoxicillin and clavulanate degradation alone and in combination in aqueous solution under frozen conditions. Int J Pharm. 2007;342(1-2):95-104. doi:10.10 16/j.jpharm.2007.05.029

15. Vahdat L, Sunderland B. The influence of potassium clavulanate on the rate of amoxicillin sodium degradation in phosphate and acetate buffers in the liquid state. Drug Dev Ind Pharm. 2009;35:471-479. doi:10.1080/03639040802438373

16. Paterson IK, Hoyle A, Ochoa G, Baker-Austin C, Taylor NGH. Optimising antibiotic usage to treat bacterial infections. Sci Rep. 2016;6:1-10. doi:10.1038/srep37853

17. Gillespie EL, Kuti JL, Nicolau DP. Pharmacodynamics of antimicrobials: treatment optimisation. Expert Opin Drug Metab Toxicol. 2005;1(3):351-361. doi:10.1517/17425255.1.3.351

18. Kambia NK, Merite N, Dine T, et al. Stability studies of amoxicillin/ clavulanic acid combination in polyolefin infusion bags. Eur J Hosp Pharm. 2010;16:30-37.

19. Nur AO, Hassaon AAA, Gadkariem EA, Osman Z, Ali GKM. Stability of co-amoxiclav reconstituted injectable solution. Eur J Pharm Med Res. 2015;2:109-123.

20. Adam D, De Visser I, Koeppe P. Pharmacokinetics of amoxicillin and clavulanic acid administered alone and in combination. Antimicrob Agents Chemother. 1982;22(3):353-357. doi:10.1128/AAC.22.3.353

been accepted for indexing on PubMed Central. The manuscript management system is completely online and includes a very quick and fair peer-review system, which is all easy to use. Visit http://www. dovepress.com/testimonials.php to read real quotes from published authors. 